\title{
Antibiotic resistance pattern in patients with urinary tract infection: an observational study
}

\author{
Rupa Devi Mallam, Usha Kiran P.*
}

Department of Pharmacology, Rangaraya Medical College, Kakinada, Andhra Pradesh, India

Received: 30 October 2019

Revised: 15 December 2019

Accepted: 16 December 2019

\author{
*Correspondence: \\ Dr. Usha Kiran P., \\ Email: ushakiranprayaga@gmail.com
}

Copyright: $(\subseteq)$ the author(s), publisher and licensee Medip Academy. This is an open-access article distributed under the terms of the Creative Commons Attribution Non-Commercial License, which permits unrestricted non-commercial use, distribution, and reproduction in any medium, provided the original work is properly cited.

\begin{abstract}
Background: Urinary tract infection (UTI) is one of the most common diseases caused by bacteria in communities and hospital settings. With the irrational prescription of antibiotics and their misuse leads to constant increase in resistance. This study aims to evaluate the spectrum and antibiotic resistance pattern of uropathogens and to provide a basis for appropriate antimicrobial therapy in patients with UTI.

Methods: A retrospective observational study was carried out for a period of 6 months from April 2018 to September 2018 at general hospital Kakinada. Data of 282 positive urine culture reports and their antibiotic susceptibility test results were collected from the records of the Microbiology department and were analyzed and depicted in percentages. Sample processing, identification of organisms, and pattern of antimicrobial sensitivity were carried out as per the Clinical and Laboratory Standards Institute guidelines.

Results: Out of 282 positive reports, Escherichia coli was the most common isolated uropathogen with a total of 148 $(52.4 \%)$ followed by Klebsiella $72(26 \%), 20$ (7.9\%) each of Citrobacter and pseudomonas aeruginosa. E. coli were resistant to nalidixic acid (59.5\%), cotrimoxazole (58.1\%), ciprofloxacin (54\%) amoxyclav (52.7\%), ampicilin $(45.9 \%)$, cefatoxime $(37.8 \%)$, cefaperazone and salbactum $(25.3 \%)$. Klebsiella were resistant to amoxyclav $(77.7 \%)$, cotrimazole (50\%), nalidixic acid (41.6\%), ampicilin (44.4\%), cefatoxime (27.7\%), ciprofloxacin (25\%). E. coli and Klebsiella were highly susceptible to nitrofurantoin, cefaperazone and salbactum, piperacillin and tazobactum, amikacin, imipenem, gentamicin.

Conclusions: The majority of the isolated bacteria were resistant to many antibiotics commonly used in clinical practices. So prior culture reports and institutional antibiograms are necessary for prescribing antibiotics rationally.
\end{abstract}

Keywords: Antibiotics, Antimicrobial resistance, Urinary tract infection

\section{INTRODUCTION}

Urinary tract infection (UTI) is one of the common bacterial infections in the human population. It is defined as presence of bacteria in urine along with urinary symptoms like dysuria, frequency, urgency and occasionally suprapubic tenderness. ${ }^{1}$ UTIs are more common in elderly than younger individuals similarly in females than males as they have short, straight and wider urethra. $^{2}$ The infection might occur at any part of the urinary tract, including urethra, bladder, ureter, renal pelvis, or renal parenchyma. The retrograde ascent of bacteria from urethra to bladder, kidney, is usually common. $^{3}$

UTI occurring in premenopausal and non-pregnant women and people with no known urological abnormalities are classified as uncomplicated, while others are considered complicated UTI. ${ }^{1}$ Untreated UTI can result in various complications such as kidney 
damage, renal scarring, and renal failure. UTI is commonly caused by Gram-negative bacteria such as Escherichia coli, Proteus species, Pseudomonas aeruginosa, Acinetobacter species, and Gram-positive bacteria such as Enterococcus species and Staphylococcus species also contribute to causing UTIs. Treatment constitutes prescription of common antibiotics. But these urinary pathogens have shown a changed pattern of susceptibility to antibiotics, resulting in an increase in resistance to commonly used antibiotics. ${ }^{5}$

The distribution of organisms and their susceptibility pattern to antibiotics vary regionally. Therefore, in-depth knowledge of the frequency of the causative microorganisms and their susceptibility to various antibiotics are necessary. It is also crucial to be aware of the changing patterns of antibiotic resistance in a locale. ${ }^{5-}$ 8 Hence, the current retrospective analysis of the uropathogens and their susceptibility pattern for six months in patients with UTI in a tertiary care hospital has been undertaken.

This study aids in facilitating the empiric treatment of patients with symptoms of UTIs, and this data would help authorities to formulate antibiotic prescription policies, at least for a region.

\section{METHODS}

An observational study was conducted for a period of 6 months from April 2018 to September 2018 in a tertiary care hospital in Kakinada. The data regarding culture and sensitivity of the organisms isolated from urine culture were collected from the records of the Microbiology Department, which included both Out-Patients (OP) and In-Patients (IP). A total of 282 positive urine cultures, non-repetitive samples during the study period were included. Sample processing, identification of organisms to the genus/species level and antimicrobial sensitivity were carried out as per the Clinical and Laboratory Standards Institute guidelines.

Antibiotics tested for sensitivity against gram-negative and gram-positive bacteria were ampicillin, amikacin, amoxycillin and clavulonic acid ,chloramphenicol, cefotaxime, ceftriaxone, ceftazidime, cefoxitin, cotrimoxozol, ciprofloxacin, ceftriaxone and sulbactum, cefaperazone and sulbactum, clindamycin, erythromycin, furazolidone, gentamycin, imipenem, kanamycin, levofloxacin, lomefloxacin, linezolid, meropenem, nitrofurantoin, norfloxacin, nalidixic acid, penicillin, piperacillin and tazobactum, teicoplanin, vancomycin. All the analysis was performed and depicted in a simple percentage method.

\section{RESULTS}

In the present study total, 580 samples were studied. Out of the 580 samples subjected to culture, $282(48.6 \%)$ were positive for growth. Among 282 cultures, 166
$(58.9 \%)$ were females, and $116(41.1 \%)$ were males (Figure 1).

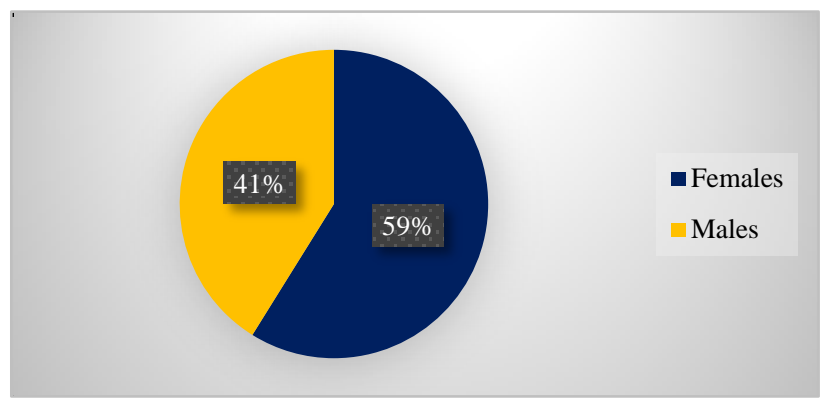

Figure 1: Age distribution.

Out of the 282 culture isolates, E.coli was the most common $148(52.4 \%)$ followed by Klebsiella $72(26 \%)$, $20(7.9 \%)$ each of Citrobacter and Pseudomonas aeruginosa, Acinetobacter 8 (2.83\%), Staph aureus 6 (2.12\%), Proteus 4 (1.41\%), Enterococcus $2(0.7 \%)$, mixed organism $2(0.7 \%)$ (Figure 2$)$.

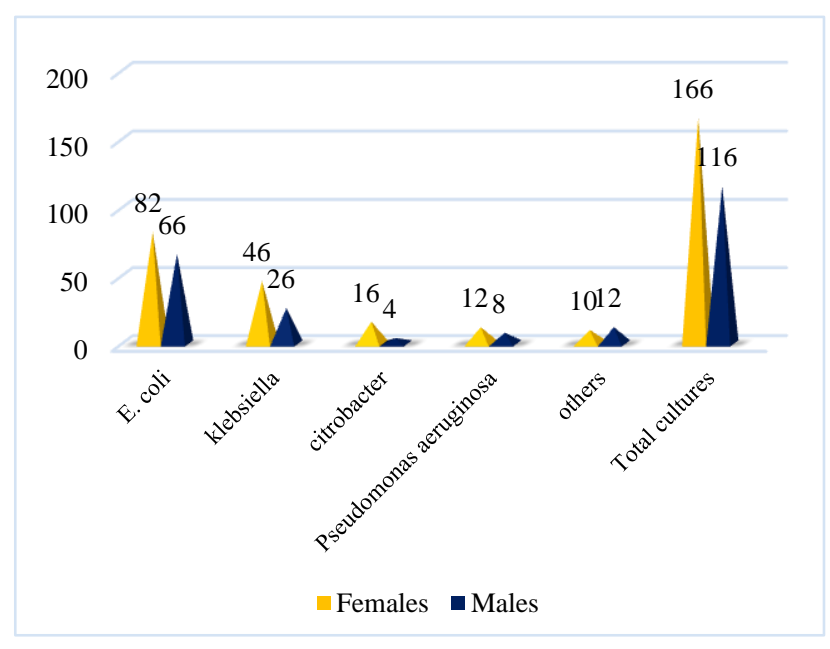

Figure 2: Organism isolation in a urine sample.

The antibiotic sensitivity and resistance pattern were analyzed for all the uropathogens (Abbreviations of antibiotics are enclosed at the end). But the sensitivity and resistance patterns for E. coli and Klebsiella depicted as they were the majority isolated uropathogens and are sorted within the Figures 3-6 in order of their sensitivity and resistance. Based on antibiotic sensitivity pattern analysis, all bacterial isolates have shown their sensitivity and resistance towards specific antibiotics.

E. coli were highly resistant to nalidixic acid (59.5\%), cotrimoxazole $(58.1 \%)$, ciprofloxacin $(54 \%)$, amoxycillin + clavulonic acid $(52.7 \%)$, ampicillin (45.9\%), cefotaxime $(37.8 \%)$, cefaperazone and sulbactum $(25.3 \%)$ (Figure 3$)$. E. coli showed high sensitivity to nitrofurantoin (72.9\%), cefaperazone and sulbactum $(64.8 \%)$, piperacillin and tazobactum $(50 \%)$, amikacin $(45.9 \%)$, imipenem $(31 \%)$, gentamycin $(30 \%)$ (Figure 4$)$. 


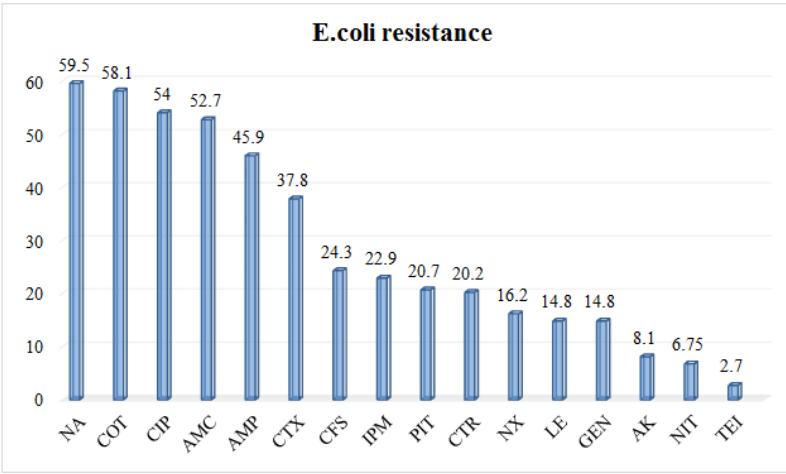

Figure 3: $E$. coli resistance pattern.

NA- nalidixic acid, COT- cotrimoxazole, CIP- ciprofloxacin, AMC- amoxicillin and clavulanic acid, AMP- ampicillin, CTXcefotaxime, CFS- cefaperazone and salbactum, IPM- imipenem, PIT- piperacillin and tazobactum, CTR- ceftriaxone, NXnorfloxacin, LE- levofloxacin, GEN- gentamycin, AKamikacin, NIT- nitrofurantoin, TEI- teicoplanin.

\section{E.coli sensitivity}

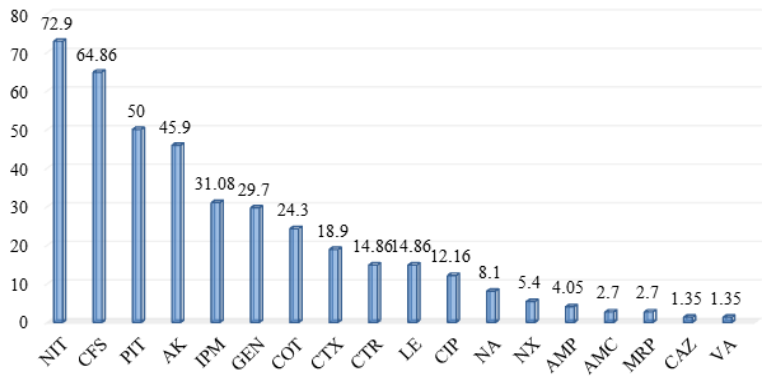

Figure 4: E. coli sensitivity pattern.

NIT- nitrofurantoin, CFS- cefaperazone and salbactum, PITpiperacillin and tazobactum, AK- amikacin, IPM- imipenem, GEN- gentamycin, , COT- cotrimoxazole , CTX- cefotaxime , CTR- ceftriaxone, LE- levofloxacin, CIP- ciprofloxacin, NAnalidixic acid, NX- norfloxacin, AMP- ampicillin, AMCamoxicillin and clavulanic acid, MRP- meropenem, CAZceftazidime, VA- vancomycin.

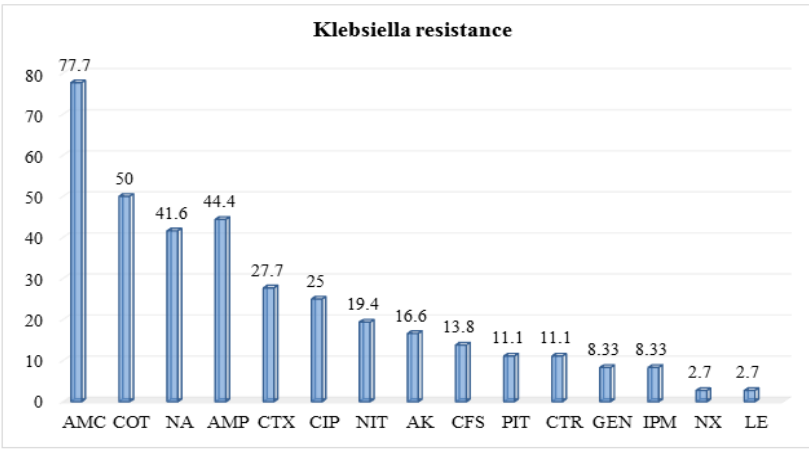

Figure 5: Klebsiella resistance pattern.

AMC- amoxicillin and clavulanic acid, COT- cotrimoxazole , NA- nalidixic acid, AMP- ampicillin, CTX- cefotaxime, CIPciprofloxacin, NIT- nitrofurantoin, AK- amikacin, CFScefaperazone and salbactum, PIT- piperacillin and tazobactum, CTR-Ceftriaxone, GEN- Gentamycin, IPM- Imipenem, NXNorfloxacin, LE- Levofloxacin.
Klebsiella were highly resistant to amoxycillin and clavulonic acid $(77.7 \%)$, cotrimoxazole $(50 \%)$, nalidixic acid (41.6\%), ampicillin (44.4\%), cefotaxime (27.7\%), ciprofloxacin (25\%) (Figure 5).

Klebsiella showed high sensitivity to cefaperazone + sulbactum (72.2\%), amikacin (58.3\%), nitrofurantoin $(55.5 \%)$, piperacillin + tazobactum $(52.7 \%)$, imipenem and cotrimoxazole (38.8\%) (Figure 6).

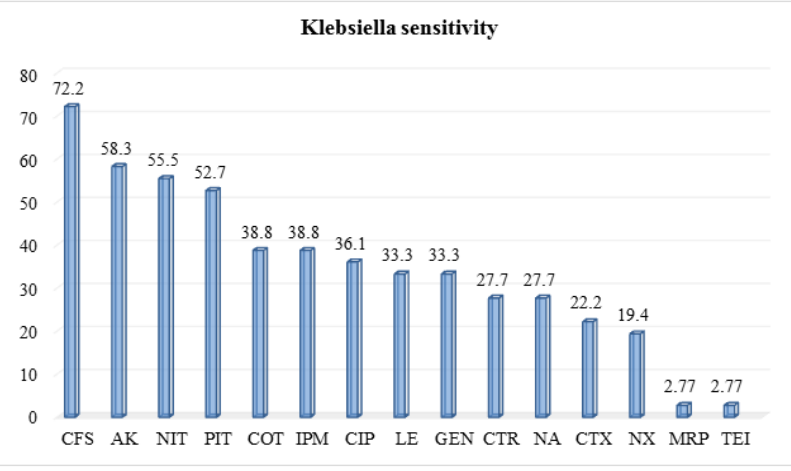

Figure 6: Klebsiella sensitivity pattern.

CFS- cefaperazone and salbactum, AK- amikacin, NITnitrofurantoin, PIT- piperacillin and tazobactum, COTcotrimoxazole , IPM- imipenem, CIP- ciprofloxacin , LElevofloxacin, GEN- gentamycin, CTR- ceftriaxone, NAnalidixic acid , CTX- cefotaxime, NX- norfloxacin,, MRPmeropenem, TEI- teicoplanin.

\section{DISCUSSION}

The current study has been conducted to evaluate the spectrum and antibiotic resistance pattern of uropathogens causing UTI and to provide a basis for appropriate antimicrobial therapy in patients with UTI. In this study, UTI was reported from $58.9 \%$ females and $48.1 \%$ males, which was similar to the findings by Bose et al, who reported UTI from $65.82 \%$ females and $34.18 \%$ males.' Female preponderance reported from various studies which were similar to this study: $(58.28 \%)$ were female and $(41.72 \%)$ were male by Rajabnia et al, $(62.42 \%)$ females and $(37.67 \%)$ males by Gupta et al, $65.82 \%$ females and $34.18 \%$ males by Bose et al. ${ }^{9-11}$

Most common organism isolated in this study were gramnegative bacteria like E. coli with $52.4 \%$ followed by Klebsiella with 26\%, 20 (7.9\%) each of Citrobacter and pseudomonas aeruginosa, Acinetobacter 8 (2.83\%), Staph aureus 6 (2.12\%), Proteus 4 (1.41\%), Enterococcus 2 $(0.7 \%)$, mixed organism $2(0.7 \%)$. The percentage of bacterial species isolated in other studies also showed similar results (Hooton et al, 1996; García-Morúa et al, 2009). ${ }^{1,9-12}$ A study conducted by Garcia-Morŭa et al, showed that $E$. coli was the commonest organism in UTI $(24.7 \%)$, followed by Candida albicans $(23.7 \%)$ among Mexican population group (Das et al). ${ }^{13,14}$ 
The sensitivity and resistance patterns of E. coli strains causing UTI vary considerably between regions and countries. Overall, gram-negative isolates showed higher resistance in the present study. This study revealed that among gram-negative bacteria, the most common strains E. coli and Klebsiella showed high resistance to commonly used empirical antibiotics like nalidixic acid, cotrimoxazole, fluoroquinolones, amoxicillin and clavulanate, cephalosporins, and other widely used antibiotics. These findings were not so similar to previous studies conducted in India, where they showed higher resistance to fluoroquinolones, cephalosporins and amoxypencillins (Akram et al and Aypak et al). ${ }^{15,16} \mathrm{~A}$ comparable result was obtained from the study conducted by Shalini et al, Amoxicillin clavulanate showed high resistance against $E$. coli $(83.3 \%)$, which is similar to the observation made by Shalini et al. ${ }^{17}$

In this current study, authors found that most $E$. coli and Klebsiella isolated in culture were susceptible to nitrofurantoin, cefoperazone-sulbactam, piperacillintazobactam, aminoglycosides (amikacin, gentamycin), imipenem and $3^{\text {rd }}$ generation cephalosporin's (cefotaxime, ceftriaxone). This pattern of antibiotic resistance has severe implications for developing countries with a more extended hospital stay and the search for more 'highpowered' and expensive antimicrobials. The resistance profile of the bacteria may be due to irrational use of antibiotics, practices of self-medication, antibiotics misuse and abuse. It can be overcome or minimized by implementing rational prescribing practices like the use of lower antibiotics with limited spectrum to which the organism is susceptible rather than resorting to prescribing higher antibiotics with a wide spectrum. ${ }^{6}$ Empirical antibiotics are prescribed for patients who present with UTI, and which should be changed once the antibiotic sensitivity pattern is available. Antibiotic stewardship programs should be initiated mainly to reduce inappropriate usage of antibiotics, reduction in costs and the reduction in the incidence of antimicrobial resistance.

Limitations of the study was included all types of UTI which could be a limitation. It would have been better if the analysis was done by differencing the UTI into complicated and uncomplicated. Data collected from records of microbiology department was written manually which can be manipulated and the other limitation was the short study period.

\section{CONCLUSION}

As the resistance to fluoroquinolones, penicillin, and cephalosporins are being increased, the feasibility of the empirical treatment of UTI with nitrofurantoin should be considered. This study fortifies the need for continuous local surveillance of bacterial antimicrobial resistance. So, prior culture reports and institutional antibiograms are necessary for prescribing antibiotics rationally. There is also a need for a comprehensive Antibiotic Stewardship
Programme in this hospital setting, while the public should be educated on the consequences of indiscriminate use of antibiotics.

\section{ACKNOWLEDGEMENTS}

Authors would like to thanks to their Professor and HOD of Pharmacology, Dr. Usha Kiran and also the faculty of the Microbiology department for their support during the study.

\section{Funding: No funding sources Conflict of interest: None declared Ethical approval: Not required}

\section{REFERENCES}

1. Maheswary D, Saikumar C. Profile of urinary tract infections and resistance patterns in a tertiary care hospital in India. Int $\mathbf{J}$ Curr Microbiol App Sci. 2018;7(3):506-12.

2. Khoshbakht R, Salimi A, Aski HS, Keshavarzi H. Antibiotic susceptibility of bacterial strains isolated from urinary tract infections in Karaj, Iran. Jundishapur J Microbiol. 2012;6(1):86-90.

3. Abejew AA, Denboba AA, Mekonnen AG. Prevalence and antibiotic resistance pattern of urinary tract bacterial infections in Dessie area, North-East Ethiopia. BMC Res Notes. 2014;7(1):687.

4. Thass N, Kumar M, Kaur R. Prevalence and antibiogram of bacterial pathogens causing urinary tract infection in a tertiary care hospital. Intern J Med Sci Pub Health. 2019;8(1):53-8.

5. Somashekara SC, Deepalaxmi S, Jagannath N, Ramesh B, Laveesh MR, Govindadas D. Retrospective analysis of antibiotic resistance pattern to urinary pathogens in a Tertiary Care Hospital in South India. J Basic Clin Pharma. 2014;5(4):105.

6. Shanmugapriya S, Saravanan T, Janani K. Antibiotic sensitivity pattern to urinary tract infections in a tertiary care hospital in South India. Int $\mathbf{J}$ Basic Clin Pharmacol. 2017;6(6):1445-50.

7. Thomas T, Tony RL, Thomas A, Santhosh SV, Gomathi M, Suresh A, et al. Antibiotic Resistance Pattern in Urinary Tract Infection during Pregnancy in South Indian Population. Asian J Pharmac. 2018;12(2):S625-30.

8. Badhan R, Singh DV, Badhan LR, Kaur A. Evaluation of bacteriological profile and antibiotic sensitivity patterns in children with urinary tract infection: A prospective study from a tertiary care center. Ind J Urol: IJU: J Urol Soci Ind. 2016;32(1):50.

9. Bose M, Basu R. Antibiotic resistance pattern of urinary isolates in a tertiary care hospital in berhampore, west Bengal. J Evolut Med Dental Sci. 2018;7(31):3466-71.

10. Rajabnia M, Bahadoram M, Fotoohi A, Mohammadi M. Antibiotic resistance patterns of urinary tract 
infections in Sanandaj, Iran $\mathbf{J}$ Nephropharmacol. 2019;8(2):26.

11. Sood S, Gupta R. Antibiotic resistance pattern of community acquired uropathogens at a tertiary care hospital in Jaipur, Rajasthan. Indian journal of community medicine: official publication of Ind Assoc Prevent Soci Med. 2012;37(1):39.

12. Hooton TM, Scholes D, Hughes JP, Winter C, Roberts PL, Stapleton AE, et al. A prospective study of risk factors for symptomatic urinary tract infection in young women. N Engl J Med. 1996;335:468-74.

13. García-Morúa A, Hernández-Torres A, Salazar-deHoyos JL, Jaime-Dávila R, Gómez-Guerra LS Community-acquired urinary tract infection etiology and antibiotic resistance in a Mexican population group. Rev Mex Urol. 2009;69(2):45-8.

14. Das RN, Chandrashekhar TS, Joshi HS, Gurung M, Shrestha N, Shivananda PG. Frequency and susceptibility profile of pathogens causing urinary tract infections at a tertiary care hospital in western Nepal. Singapore Med J. 2006;47(4):281.
15. Akram M, Shahid M, Khan AU. Etiology and antibiotic resistance patterns of community-acquired urinary tract infections in JNMC Hospital Aligarh, India. Annals Clin Microbiol Antimicrob. 2007;6(1):4.

16. Aypak C, Altunsoy A, Düzgün N. Empiric antibiotic therapy in acute uncomplicated urinary tract infections and fluoroquinolone resistance: a prospective observational study. Annal Clin microbiol Antimicrob. 2009;8(1):27.

17. Joshi MC, Rashid MK, Joshi HS. Study of antibiotic sensitivity pattern in urinary tract infection at a tertiary hospital. Natl J Integr Res Med. 2011;2:43-6.

Cite this article as: Mallam RD, Kiran UP. Antibiotic resistance pattern in patients with urinary tract infection: an observational study. Int J Basic Clin Pharmacol 2020;9:195-9. 